\title{
Assessment Relativism and the Truth-Predicate
}

\author{
Henrik Sova \\ University of Tartu \\ Ülikooli 18, \\ Tartu 50090, Estonia \\ Email: henrik.sova@gmail.com
}

\begin{abstract}
The purpose of this paper is to argue that assessment relativism entails the assessment-sensitivity of the sentential truth-predicate, but not of the propositional truth-predicate. The central idea of assessment relativism is that a single token claim evaluated within a single world can have different truth-values when considered in different contexts of assessment. John MacFarlane in Assessment Sensitivity: Relative Truth and its Applications (2014) and also Max Kölbel in the article 'Global relativism and self-refutation' (2011) have argued that this position leads to relativism about the propositional truth-predicate. I argue that this is not the case-it entails relativism only about the sentential truth-predicate.
\end{abstract}

Keywords: assessment relativism, context of assessment, relativism, truthpredicate

\section{Introduction}

The purpose of this paper is to argue that assessment relativism entails the assessment sensitivity of the sentential truth-predicate, but not of the propositional truth-predicate. The central idea of assessment relativism is that a single token claim evaluated within a single world can have different truthvalues when considered in different contexts of assessment. It has been suggested that this position leads to relativism about the propositional truth-predicate (see Kölbel, 2011, p. 20; MacFarlane, 2014, p. 93). I argue that this is not the caseit entails relativism only about the sentential truth-predicate. 
Among the contemporary analytic accounts of relativism, the most developed version of assessment relativism has been put forward by John MacFarlane (2005; 2014). Thus, I take his account to be the paradigm case of assessment relativism, in the context of which the arguments advanced here should be considered. In his works, MacFarlane has introduced a new parameter for truth-context of assessment-in addition to two more familiar indexes, context of utterance (Lewis, 1980) and circumstance of evaluation (Kaplan, 1989). In short, a context of assessment is a possible situation in which a use of sentence or proposition might be assessed in terms of its truth-value (MacFarlane, 2014, p. 60). Assessment relativism for some languages holds that at least some sentences or propositions in that language are assessment-sensitive in the sense that their truth-values depend on the context of assessment (see MacFarlane, 2005, p. 310; 2014 , p. 65). MacFarlane (2005, p. 309; 2014, p. 60) argues that only this kind of relativism is proper relativism.

Depending on the discourse at hand, there are a variety of possible assessmentcontextual parameters (see Wright, 2008, p. 381 for an overview). For example, a relativist about the discourse of aesthetics could take one relevant parameter to be the aesthetic standard of the assessor. The sentence "the Mona Lisa is beautiful", as used in a given context, can then be assessed as true or false according to the aesthetic standard of the person who assesses the sentence as used in that context (see MacFarlane, 2014, pp. 88-91). Correspondingly, the predicate "is beautiful" would then be assessment-sensitive, because its extension would depend on a feature of the context of assessment-namely, the assessor's aesthetic standard.

This paper addresses the question whether in an assessment-sensitive language the truth-predicate "is true" must be assessment-sensitive, too. The paper proceeds as follows. In the first section, I will explicate the argument that John MacFarlane and Max Kölbel put forth for the conclusion that assessment relativism entails the assessment sensitivity of the propositional truth-predicate. The explication makes it evident that the argument is not valid. In the second section, I will show how assessment relativism entails the assessment sensitivity of the sentential truth-predicate. Hence, I conclude that if a language is assessment-sensitive, then we are committed to relativism about the sentential truth-predicate of that language, but not to relativism about the propositional truth-predicate. This paper thus shows that in considering how a relativist position in one area of discourse affects a discourse regarding truth, it is crucial to distinguish between these two types of truth-predicates. 


\section{Assessment relativism does not entail the assessment sensitivity of the propositional truth-predicate}

In this section, I argue that assessment relativism does not entail the assessment sensitivity of the propositional truth-predicate, even though John MacFarlane and Max Kölbel have claimed otherwise. I begin by explaining the concept of propositional truth-predicate and then explicate the argument of MacFarlane and Kölbel in the context of assessment relativism. The explication reveals that their argument is not valid.

In general, when a predicate $F$ is applied to an argument $a$, it yields a formula $F a$ that attributes the property that is the semantic value of $F$ to the entity that is the semantic value of $a$. The propositional truth-predicate attributes the property of being true to a proposition in a given circumstance of evaluation and context of assessment (such that the proposition is apt for a certain truthvalue). Since a proposition is the semantic value of a sentence, the argument that the propositional truth-predicate is applied to is a sentence (or a formula, in more formal contexts). Thus, more formally put, when the propositional truthpredicate $\mathrm{T}_{\mathrm{P}}$ is applied to sentence $\Phi$ in a given context, it yields a formula $\mathrm{T}_{\mathrm{P}} \Phi$ that attributes the property that is the semantic value of $T_{p}$-i.e., truth-to the semantic value of $\Phi$, which is a proposition. In formal semantic terms, the propositional truth-predicate is a predicate in the standard sense of an expression of type $(\mathrm{x}, \mathrm{t})$. That is, a predicate is an expression that, when applied to an expression of type $\mathrm{x}$ (where $\mathrm{x}$ may be any type), it yields an expression of type $\mathrm{t}$ (a formula). Specifically, the propositional truth-predicate is applied to formulas and thus its type is $(t, t)$. The semantic value of this type of an expression is a function from propositions (with a certain truth-value) to propositions (with a certain truth-value). The propositional truth-predicate expresses a function which yields a true proposition if the input is a true proposition and a false proposition if the input is a false proposition.

Also note that, in the current context, the truth-predicate does not attribute the property of being true to the propositional content in the sense that the latter is a function from context to truth-value. There are two reasons for that. First, in standard formal semantics, a truth-predicate does not operate on a function from context to truth-value. It operates either on formulas (a propositional truthpredicate) or individuals (a sentential truth-predicate, see Section 2). Second, MacFarlane does not interpret propositions in terms of a propositional content in the sense of a function from a possible world and context of assessment to 
a truth-value. MacFarlane (2014, pp. 71-72) takes propositions to be abstract objects, the truth of which we might disagree on. In contrast, a function from a possible world and context of assessment to a truth-value is not something we disagree on in a case in which we disagree whether a proposition is true. For MacFarlane, propositions are abstract objects that have a truth-value (expressed by the expression of type $\langle t\rangle)$ and they can be assessment-sensitive. They are abstract objects to which the propositional truth-predicate attributes the property of being true. They are also objects that the equivalence schema is applied to (to which we will turn shortly).

Natural languages have a variety of ways for expressing the property behind the propositional truth predicate. For example:

(1) It is true that the Mona Lisa is beautiful

and

(2) The proposition that the Mona Lisa is beautiful is true.

Put into terms of lambda calculus, the predicate in (1) that expresses truth is " $(\lambda \Phi)$ (It is true that $\Phi)$ "; in (2), it is " $(\lambda \Phi)$ (The proposition that $\Phi$ is true)". We can think of (1) and (2) as resulting from the application of these predicates to the sentence "the Mona Lisa is beautiful".

Before turning to the argument of MacFarlane and Kölbel, let me first introduce some further notation. Let $c$ be a variable for all the parameters that are relevant for determining the extension of the expression (such as the context of utterance or circumstance of evaluation) except the context of assessment. Let $a$ be a variable for the context of assessment. Let $\llbracket E \rrbracket^{c, a}$ stand for the extension of the expression $\mathrm{E}$ with respect to the configuration of parameters given by $c$ and a. Given this notation, expression $\mathrm{E}$ in a language is assessment-sensitive if its extension changes across different contexts of assessment while other parameters of truth remain constant - that is, for some $c, a$ and $a^{\prime}$ :

$$
\llbracket \mathrm{E} \rrbracket^{\mathrm{c}, \mathrm{a}} \neq \llbracket \mathrm{E} \rrbracket^{\mathrm{c}, \mathrm{a}^{\prime}}
$$

Assessment relativism for some languages holds that at least some sentences in that language are assessment-sensitive in this sense (see MacFarlane, 2005, p. 310; 2014, p. 65).

The argument that MacFarlane and Kölbel put forth is the following. If assessment relativism is true for some language-meaning that it contains some assessment-sensitive sentences — and if the propositional truth-predicate of that 
language is governed by the equivalence schema, then it follows that the truthpredicate of that language must be assessment-sensitive. MacFarlane expresses the argument as follows: "[I]f the language can express any assessment-sensitive propositions, "true" will also be assessment-sensitive, since if $p$ is assessmentsensitive, the proposition that $p$ is true must be assessment-sensitive too" (MacFarlane, 2014, p. 93). Kölbel puts the same point in more general terms, claiming that if propositional truth is subject to the equivalence schema, then relativism about any feature entails relativism about the feature of truth itself (Kölbel, 2011, pp. 17-20). ${ }^{1}$

The explication of their argument in more precise terms is as follows. Let $\Phi_{1}$ be some assessment-sensitive sentence such that for some $c, a$ and $a^{\prime}$ :

(1) $\llbracket \Phi_{1} \rrbracket^{c, a} \neq \llbracket \Phi_{1} \rrbracket^{c, a^{\prime}}$

The equivalence schema for the propositional truth-predicate states that for any c,a-pair ${ }^{2}$ :

(ES PROP) $\llbracket \mathrm{T}_{\mathrm{P}} \Phi \leftrightarrow \Phi \rrbracket^{\mathrm{c}, \mathrm{a}}=$ True

MacFarlane and Kölbel argue that ES PROP and (1) entail the assessment sensitivity of the propositional truth-predicate $T_{\mathrm{P}}$ :

(2) $\llbracket \mathrm{T}_{\mathrm{p}} \rrbracket^{\mathrm{c}, \mathrm{a}} \neq \llbracket \mathrm{T}_{\mathrm{p}} \rrbracket^{\mathrm{c}, \mathrm{a}^{\prime}}$

However, this explication makes it evident that, contra MacFarlane and Kölbel, ES PROP and (1) do not entail (2). From (1) and the equivalence stated in ES PROP, it only follows that $\mathrm{T}_{\mathrm{P}} \Phi_{1}$ as a whole must be assessment-sensitive. That is, the truth-value of $T_{P} \Phi_{1}$ indeed changes due to switching from the context of assessment a to the context a':

$$
\llbracket \mathrm{T}_{\mathrm{P}} \Phi_{1} \rrbracket^{c, a} \neq \llbracket \mathrm{T}_{\mathrm{P}} \Phi_{1} \rrbracket^{\mathrm{c}, \mathrm{a}^{\prime}}
$$

But this conclusion falls short of establishing the assessment sensitivity of the propositional truth-predicate $\mathrm{T}_{\mathrm{p}}$. According to the premise (1), the constituent $\Phi_{1}$

1 According to Kölbel (2011, p. 16), relativism about a feature $F$ means that some object has a feature $F$ relative to one value of a parameter $P$ and not relative to another value of $P$. In the context of assessment relativism, values of parameter $P$ are particular contexts of assessments. Thus, as an instance of his general claim, Kölbel argues that if propositional truth is subject to the equivalence schema, then assessment sensitivity of any feature entails the assessment sensitivity of the feature propositional truth, which is represented in a language as the assessment sensitivity of the propositional truth-predicate.

2 It is uncontroversial to assume that the equivalence schema holds in every context. 
in $T_{\mathrm{p}} \Phi_{1}$ is already assessment-sensitive and this suffices to explain the assessment sensitivity of $T_{p} \Phi_{1}$. Therefore, we have not proven that the predicate $T_{p}$ must be assessment-sensitive too. In sum, although MacFarlane is right to point out that "if $p$ is assessment-sensitive, the proposition that $p$ is true must be assessmentsensitive too" (MacFarlane, 2014, p. 93), it does not follow that it is namely the propositional truth-predicate that must be assessment-sensitive.

\section{Assessment relativism entails the assessment sensitivity of the sentential truth-predicate}

In this section, I argue that assessment relativism entails the assessment sensitivity of the sentential truth-predicate. In the case of sentential truth-predicate, the argument that the predicate is applied to is a quotation-name of a sentence (that is, a name constructed by placing quotation marks around a sentence). The semantic value of a quotation-name of a sentence is a sentence. Thus, in short, the sentential truth-predicate applies to the quotation-names of sentences and attributes the property of being true to sentences. In formal semantic terms, the sentential truthpredicate is of the semantic type $\langle e, t\rangle$, denoting a function from individuals to propositions with a truth-value. The domain of individuals in this case is the set of sentences, denoted by singular terms, i.e., the quotation-names of sentences. In natural language, an example of applying the sentential truth-predicate would be the following claim: the sentence "the Mona Lisa is beautiful" is true. ${ }^{3}$

The conclusion that assessment relativism entails the assessment sensitivity of the sentential truth-predicate follows from two acceptable premises. The first is that the denotation of quotation-names does not change from one context to another. The name denotes the same sentence in every context. ${ }^{4}$ For example, the quotation-name "the Mona Lisa is beautiful" denotes (or mentions or refers to)

3 Since sentences express propositions, one can perhaps take the semantic value of a sentence's quotation-name to be a proposition. Although the idea that a singular term could have the same semantic value as a sentence (that is, a proposition) is controversial, I just note that the following argument for the assessment sensitivity of the sentential truth-predicate does not depend on whether we interpret the quotation-name as referring to a sentence or to a proposition expressed by the sentence. That is, the argument is not affected by whether we consider names of sentences or names of propositions. Thus, if we think of the truth predicate as requiring a nominalisation of propositions or propositional content, then in the current context we effectively mean the type $\langle e, t\rangle$ truth-predicate.

4 Some philosophers hold that quotation always involves indexicality (see Burge, 1978; Davidson, 1968). However, my argument goes through even if the extension of quotation-names varies with context of use, but remains constant in different contexts of assessment. 
the same sentence when considered in different contexts of assessment. In other words, quotation-names are assessment-insensitive. Let each sentence $\Phi$ have a quotation-name ${ }^{\ulcorner} \Phi^{\top}$. The first premise then states that for any $c, a$-pair:

$$
\text { (QN) } \llbracket^{r} \Phi^{\top} \rrbracket^{\mathrm{c}, \mathrm{a}}=\Phi
$$

The second premise is that the sentential truth-predicate is governed by the equivalence schema in every context. Thus, letting $T_{S}$ be a sentential truthpredicate, the premise states that for any $c, a$-pair:

$$
\text { (ES SENT) } \llbracket \mathrm{T}_{S}\left({ }^{r} \Phi^{\top}\right) \leftrightarrow \Phi \rrbracket^{c, a}=\text { True }^{5}
$$

Premises QN and ES SENT are sufficient for showing that assessment relativism for a language entails the assessment sensitivity of the sentential truth-predicate in that language. Recall that assessment relativism for a language means that the language contains at least some assessment-sensitive sentences. Again, let $\Phi_{1}$ be some assessment-sensitive sentence such that for some $c, a$ and $a^{\prime}$ :

$$
\llbracket \Phi_{1} \rrbracket^{c, a} \neq \llbracket \Phi_{1} \rrbracket^{c, a^{\prime}}
$$

Analogously to the case of propositional truth in the previous section, we can observe that given (1) and ES SENT, the left-hand side of the equivalence in ES SENT must also change its truth value due to the shift in context of assessment. In other words, it follows from (1) and ES SENT that $T_{S}\left({ }^{r} \Phi_{1}{ }^{\top}\right)$ must be assessment-sensitive:

$$
\llbracket \mathrm{T}_{S}\left({ }^{\mathrm{r}} \Phi_{1}{ }^{\top}\right) \rrbracket^{\mathrm{c}, \mathrm{a}} \neq \llbracket \mathrm{T}_{S}\left({ }^{r} \Phi_{1}{ }^{\top}\right) \rrbracket^{\mathrm{c}, \mathrm{a}}
$$

According to QN, however, the sentence mentioned by ${ }^{ } \Phi_{1}{ }^{7}$ remains constant in every $c, a$-pair and thus, ${ }^{r} \Phi_{1}{ }^{\top}$ is not assessment-sensitive. Therefore, the only constituent in $T_{S}\left({ }^{r} \Phi_{1}{ }^{\top}\right)$ that could be responsible for its assessment sensitivity must be the sentential truth-predicate $\mathrm{T}_{S}$. Thus, $\mathrm{T}_{S}$ is an assessment-sensitive predicate - meaning that for some $c, a$ and $a^{\prime}$ :

$$
\llbracket \mathrm{T}_{S} \rrbracket^{c, a} \neq \llbracket \mathrm{T}_{S} \rrbracket^{c, a^{\prime}}
$$

In sum, given QN and ES SENT, assessment relativism about a language entails that the sentential truth-predicate of that language must also be assessmentsensitive.

5 I assume sufficient restrictions on the equivalence schema to avoid semantic paradoxes. I also assume that metalanguage contains object language, so that we can straightforwardly construct a quotation-name of a sentence by using quotation marks. 
The parallel argument for the assessment sensitivity of the propositional truthpredicate fails because of a crucial difference between the propositional truthpredicate and sentential truth-predicate-the former applies to sentences that express propositions (i.e., its semantic type is $\langle t, t\rangle)$, while the latter applies to quotation-names of sentences (i.e., its semantic type is $\langle e, t\rangle)$. Thus the issue is about whether we consider type $\langle t, t\rangle$ or type $\langle e, t\rangle$ truth-predicate in the equivalence schema. Both options are legitimate. Since it is reasonable to assume that the denotation of quotation-names does not change from one context to another, this difference is sufficient to establish that in an assessment-sensitive language, the sentential truth-predicate must be assessment-sensitive too, while the propositional one might be not.

For a concluding illustration, let us assume that the sentence "the Mona Lisa is beautiful" is assessment-sensitive in our language. If we now apply propositional truth to this sentence while assuming the equivalence schema, then it follows that the sentence "It is true that the Mona Lisa is beautiful" is also assessmentsensitive. However, for the assessment sensitivity of the latter sentence, it suffices to hold that the sentence "the Mona Lisa is beautiful" is assessment-sensitive, which we already assumed. Hence, we cannot prove that the propositional truth-predicate must be assessment-sensitive too. In the case of the sentential truth-predicate, however, we apply the predicate to the name of the assessmentsensitive sentence "the Mona Lisa is beautiful". Given the equivalence schema, it follows that the sentence " "the Mona Lisa is beautiful" is true" is assessmentsensitive. Since the sentence mentioned by the quotation-name denotes the same sentence in every context, we can conclude that the assessment sensitivity originates namely from the sentential truth-predicate "is true".

\section{Acknowledgments}

This research was supported by the University of Tartu ASTRA Project PER ASPERA and the Centre of Excellence in Estonian Studies (European Regional Development Fund), and is related to the research project IUT20-5 (Estonian Ministry of Education and Research). 


\section{References}

Burge, T. (1978), 'Self-reference and translation,' in M. Guenther-Reutte \& F. Guenther (eds.) Translation and Meaning, London: Duckworth \& Company.

Davidson, D. (1968), 'On saying that,' Synthese, vol. 19, no. 1/2, pp. 130-146. https://doi.org/10.1007/BF00568054

Kaplan, D. (1989), 'Demonstratives,' in J. Almog, J. Perry \& H. Wettstein (eds.) Themes from Kaplan, Oxford: Oxford University Press, pp. 481-563.

Kölbel, M. (2011), 'Global relativism and self-refutation,' in S. D. Hales (ed.) A Companion to Relativism, Oxford: Wiley-Blackwell, pp. 9-30. https://doi.org/10.1002/9781444392494.ch1

Lewis, D. (1980), 'Index, context, and content,' in S. Kanger \& S. Öhman (eds.) Philosophy and Grammar, Dordrecht: Reidel, pp. 79-100. https://doi.org/10.1007/978-94-009-9012-8_6

MacFarlane, J. (2005), 'Making sense of relative truth,' Proceedings of the Aristotelian Society, vol. 105, no. 3, pp. 321-339. https://doi.org/10.1111/j.0066-7373.2004.00116.x

MacFarlane, J. (2014), Assessment Sensitivity: Relative Truth and its Applications, Oxford: Clarendon Press. https://doi.org/10.1093/acprof:oso/9780199682751.001.0001

Wright, C. (2008), 'Fear of relativism?' Philosophical Studies, vol. 141, no. 3, pp. 379-390. https://doi.org/10.1007/s11098-008-9280-7

Dr Henrik Sova earned his PhD in philosophy from the University of Tartu in 2021, the title of the thesis was The Structure of a Consistent Global Pragmatism. His main research interests are contemporary pragmatism and the philosophy of Ludwig Wittgenstein, Richard Rorty, Robert Brandom and Huw Price. 